NOTICE: this is the author's version of a work that was accepted for publication in Systems \& Control Letters. Changes resulting from the publishing process, such as peer review, editing, corrections, structural formatting, and other quality control mechanisms may not be reflected in this document. Changes may have been made to this work since it was submitted for publication. A definitive version was subsequently published in Systems \& Control Letters [VOL59, ISSUE2, 2010] http://dx.doi.org/10.1016/j.sysconle.2009.12.006 


\title{
On the solution of the Riccati differential equation arising from the LQ optimal control problem*
}

\author{
Lorenzo Ntogramatzidis $^{\star} \quad$ Augusto Ferrante $^{\dagger}$ \\ ${ }^{\star}$ Dipartment of Mathematics and Statistics, \\ Curtin University of Technology, Perth, 6845 WA, Australia \\ L.Ntogramatzidis@curtin.edu.au \\ †Dipartimento di Ingegneria dell'Informazione, \\ Università di Padova, via Gradenigo, 6/B - 35131 Padova, Italy \\ augusto@dei.unipd.it
}

\begin{abstract}
In this paper we consider the matrix Riccati differential equation (RDE) that arises from linear-quadratic (LQ) optimal control problems. In particular, we establish explicit closed formulae for the solution of the RDE with a terminal condition using particular solutions of the associated algebraic Riccati equation. We discuss how these formulae change as assumptions are progressively weakened. An application to LQ optimal control is briefly analysed.
\end{abstract}

Keywords: Riccati differential equation, LQ optimal control, Hamiltonian differential equation, extreme solutions of the algebraic Riccati equation, sign-controllability.

*This work was partially supported by the Australian Research Council (Discovery Grant DP0986577) and by the Italian Ministry for Education and Resarch (MIUR) under PRIN grant "Identification and Robust Control of Industrial Systems". 


\section{Introduction}

The matrix differential Riccati equation is uniquitous in systems and optimal control/filtering theory and, for this reason, it has always received a great deal of attention, see e.g. [2] and the bibliography therein. For example, the solution of the classical finite-horizon linear-quadratic (LQ) optimal control problem is traditionally expressed in terms of the solution of a matrix Riccati differential equation with a terminal condition (see for example the classic textbooks $[1,23])$.

From the Seventies, a significant stream of literature emerged regarding the problem of devising numerically reliable and efficient algorithms for the integration of the Riccati differential equation, see e.g. $[10,18]$. Some of these methods, that make use of the so-called Bernoulli substitution technique, consist in exploiting the solution of a larger linear differential equation (the so-called Hamiltonian differential equation), whose size is twice the size of the Riccati equation to be solved. Another important class of algorithms is based on the so-called Chandrasekhar decomposition [21, 18], whereby the solution of the Riccati differential equation is obtained by solving a pair of coupled matrix linear differential equations. Other important approaches aimed at solving the so-called periodic differential Riccati equation include the multi-shot and iterative algorithms, see [31, 17,30] and [11], respectively. See also [2].

Another area of intense research activity, which originated in the early Eighties and flourished in the Nineties, was centered on the attempt to characterise the solutions of the differential Riccati equation - particularly in the linear time-invariant (LTI) case - by using non-recursive formulae. The first and most general formula introduced in the literature exploits the solution of the Hamiltonian differential equation via a Bernoulli substitution as described above. However, this formula was soon found to be unsuitable for studying important properties of the solutions of the Riccati differential equation, such as finite escape times, convergence and mechanisms of attraction. Another drawback associated with Bernoulli substitutions is the difficulty that arises in determining an expression for the variations of the solution of the Riccati differential equation as an explicit function of variations of some parameters of the problem. Therefore, over the last twenty years, many researchers have focused their attention to finding alternative closed-form representations of the solution of the Riccati differential equation, [6, 5, 25]. Most of the representations proposed so far in the literature exploit the extreme (i.e., stabilising and anti-stabilising) solutions of the associated algebraic Riccati equation. These representations can be used to study the dependence of the solution of the Riccati differential equation on certain parameters of the problem in the cases when the functional dependence of the solution of the associated algebraic Riccati equation on such parameters is computable (as it happens, for example, in $[7,8])$. The problem associated with these alternative representations is that the 
extreme solutions of the algebraic Riccati equation both exist only if the underlying system (in the case of the Riccati equation arising from the finite-horizon LQ problem) is controllable.

The aim of this paper is to obtain more general representations for the solution of the Riccati differential equation, by progressively removing the assumptions on the problem data. We begin by considering a formula that is valid under the very mild assumption of sign-controllability, $[29,28]$ : this is the weakest form of controllability introduced so far in the literature, as it encompasses controllability, stabilisability and anti-stabilisability. Moreover, sign-controllability is also the weakest known assumption for which the associated algebraic Riccati equation is guaranteed to admit a symmetric solution. Under this assumption, a formula parameterising in finite terms all the trajectories originating from the Hamiltonian differential equation, introduced by the same authors in [14], is exploited to derive a non-recursive formula for the solution of the Riccati differential equation. This parameterisation of the trajectories of the Hamiltonian differential equation generalises those proposed in [15] and [27] for controllable and stabilisable systems, respectively; see also $[13,12]$ for the discrete-time counterpart. In the particular case of stabilisability, it is also shown that using the Kalman controllability decomposition it is possible to reduce the size of the algebraic Riccati equation to be solved. Furthermore, particular cases are also identified where a solution of the algebraic Riccati equation exists even when the sign-controllability assumption does not hold. This task is accomplished by introducing a first new sign-controllability form, that gives a sign-controllable part whose dimension is the largest that can be obtained. In the second part of the paper, the sign-controllability assumption is relaxed further, and a second sign-controllability form is explioted to the end of employing a solution of an algebraic Riccati equation to parameterise the trajectories of the sole signcontrollable part of the Hamiltonian differential equation, while the nonsign-controllable part is handled using the aforementioned Bernoulli substitution method. In this way, it is proven that if the nonsign-controllable part of the system has no common modes with the sign-controllable part of the Hamiltonian matrix, and such sign-controllable part has no modes on the imaginary axis, the solution of the Riccati differential equation can still be represented in a non-recursive fashion. In the last part of the paper, the applications of these results to optimal control problems are discussed. In particular, we show that using an approach that is similar in spirit to that proposed in $[15,24,27,14]$ very general linear-quadratic optimal control problems with affine constraints on the state vector at the end-points and with the most general type of quadratic performance index can be solved in closed form. 


\section{Problem formulation}

In this paper we consider the problem of finding closed-form representations for the solutions $P(t), t \in[0, T]$ of the matrix Riccati differential equation

$$
\dot{P}(t)+A^{\top} P(t)+P(t) A-(P(t) B+S) R^{-1}(P(t) B+S)^{\top}+Q=0
$$

with terminal condition

$$
P(T)=P_{T}
$$

where $T>0, A \in \mathbb{R}^{n \times n}, B \in \mathbb{R}^{n \times m}$, with $m \leq n$; the matrices $Q \in \mathbb{R}^{n \times n}, S \in \mathbb{R}^{n \times m}$ and $R \in \mathbb{R}^{m \times m}$ are such that the so-called Popov matrix

$$
\Pi:=\left[\begin{array}{cc}
Q & S \\
S^{\top} & R
\end{array}\right]
$$

is symmetric and positive semidefinite, and $R$ is positive definite; finally, the terminal condition $P_{T} \in \mathbb{R}^{n \times n}$ is symmetric and positive semidefinite.

We recall that the Popov triple is defined as the triple $\Sigma:=(A, B, \Pi)$, see e.g. [16].

The matrix Riccati differential equation (1) is concisely denoted by $\operatorname{RDE}(\Sigma)$, while the differential problem (1-2) is referred to as the Riccati differential problem and denoted by $\operatorname{RDP}\left(\Sigma, P_{T}\right)$. The solution of $\operatorname{RDE}(\Sigma)$ and $\operatorname{RDP}\left(\Sigma, P_{T}\right)$ are related to the solution $X, \Lambda$ : $(-\infty, T] \longrightarrow \mathbb{R}^{n \times n}$ of the Hamiltonian differential equation $\operatorname{HDE}(\Sigma)$

$$
\left[\begin{array}{c}
\dot{X}(t) \\
\dot{\Lambda}(t)
\end{array}\right]=H\left[\begin{array}{c}
X(t) \\
\Lambda(t)
\end{array}\right], \quad \text { where } \quad H=\left[\begin{array}{cc}
A-B R^{-1} S^{\top} & -B R^{-1} B^{\top} \\
-Q+S R^{-1} S^{\top} & -A^{\top}+S R^{-1} B^{\top}
\end{array}\right],
$$

and to the solution of the Hamiltonian differential problem $\operatorname{HDP}\left(\Sigma, P_{T}\right)$, i.e., a solution $X(t), \Lambda(t)$ of $\operatorname{HDE}(\Sigma)$ that also satisfies the boundary condition

$$
\left[\begin{array}{l}
X(T) \\
\Lambda(T)
\end{array}\right]=\left[\begin{array}{l}
I_{n} \\
P_{T}
\end{array}\right]
$$

The relation between the solutions of $\operatorname{RDE}(\Sigma)$ and $\operatorname{RDP}\left(\Sigma, P_{T}\right)$ with those of $\operatorname{HDE}(\Sigma)$ and $\operatorname{HDP}\left(\Sigma, P_{T}\right)$ is stated in precise terms as follows.

Theorem 1 ([9], pp. 274-275) Let $X, \Lambda:(-\infty, T] \longrightarrow \mathbb{R}^{n \times n}$ be the solutions of the Hamiltonian differential problem $\operatorname{HDP}\left(\Sigma, P_{T}\right)$. Then

1. $X(t)$ is non-singular for all $t \in(-\infty, T]$;

2. the solution $P(t)$ of the $\operatorname{RDP}\left(\Sigma, P_{T}\right)$ is

$$
P(t)=\Lambda(t) X^{-1}(t), \quad t \in(-\infty, T] .
$$


Moreover, if $P(t)$ is a solution of the $\operatorname{RDE}(\Sigma)$ on $(-\infty, T]$ and $X(t):(-\infty, T] \longrightarrow \mathbb{R}^{n \times n}$ is a solution of the matrix differential equation

$$
\dot{X}(t)=\left(A-B R^{-1}\left(B^{\top} P(t)+S^{\top}\right)\right) X(t)
$$

then $X(t)$ and $\Lambda(t)=P(t) X(t)$ are a solution of $\operatorname{HDE}(\Sigma)$.

Theorem 1 provides a first general way to characterise the solution of both $\operatorname{RDE}(\Sigma)$ and $\operatorname{RDP}\left(\Sigma, P_{T}\right)$. Indeed, linearity of $\operatorname{HDE}(\Sigma)$ yields

$$
\left[\begin{array}{c}
X(t) \\
\Lambda(t)
\end{array}\right]=e^{H t} C
$$

where $C \in \mathbb{R}^{2 n \times n}$ is an integration constant that can be found by imposing the boundary condition (4) on (6). As such, the solution of $\operatorname{HDP}\left(\Sigma, P_{T}\right)$ is given by

$$
\left[\begin{array}{c}
X(t) \\
\Lambda(t)
\end{array}\right]=e^{H(t-T)}\left[\begin{array}{c}
I \\
P_{T}
\end{array}\right] .
$$

If we partition the exponential matrix $e^{H(t-T)}$ as $\left[\begin{array}{ll}E_{1}(t) & E_{2}(t) \\ E_{3}(t) & E_{4}(t)\end{array}\right]$, where each $E_{i}(t)$ is an $n \times n$ time varying matrix, we can express $X(t)$ and $\Lambda(t)$ as $X(t)=E_{1}(t)+E_{2}(t) P_{T}$ and $\Lambda(t)=$ $E_{3}(t)+E_{4}(t) P_{T}$, which enable the solution of $\operatorname{RDP}\left(\Sigma, P_{T}\right)$ to be written as

$$
P(t)=\Lambda(t) X^{-1}(t)=\left(E_{3}(t)+E_{4}(t) P_{T}\right)\left(E_{1}(t)+E_{1}(t) P_{T}\right)^{-1} .
$$

This approach for solving the Riccati differential equation is known in the literature as Bernoulli substitution, [18, 25], and constitutes the basis of the so-called Davison-Maki numerical method, [10]. Expression (7) has the advantage of being very general. On the other hand, since there is no explicit way to express the submatrices $E_{i}(t)$ as a function of the problem data $\Sigma$, this formula is not suitable for studying properties like convergence, limiting behaviour of the solution and mechanisms of attraction, [6, 5]. Moreover, (7) does not allow to analyse how the solution varies in terms of perturbation of $\Sigma$ or $P_{T}$, which is a fundamental question in many practical and theoretical problems. For this reason, remarkable efforts have been devoted to finding more explicit representations for the solutions of the Riccati differential equation, see e.g. [5] and the references therein. In this paper, we present new ways to represent the solution of the Riccati differential problem under mild assumptions that are progressively weakened, and generalise the ones previously introduced in the literature in several directions. 


\section{Closed-form solutions under Sign-Controllability assump- tion}

\subsection{Background material}

Consider the continuous-time algebraic Riccati equation $\operatorname{CARE}(\Sigma)$

$$
P A+A^{\top} P-(S+P B) R^{-1}\left(S^{\top}+B^{\top} P\right)+Q=0 .
$$

To any solution $P=P^{\top} \in \mathbb{R}^{n \times n}$ of $\operatorname{CARE}(\Sigma)$ corresponds the closed-loop matrix

$$
A_{P}:=A-B R^{-1}\left(S^{\top}+B^{\top} P\right) .
$$

The set of eigenvalues $\sigma\left(A_{P}\right)$ of $A_{P}$ is a subset of the spectrum of the Hamiltonian matrix $H$, [26, Theorem 6]. The following esistence properties of solutions to algebraic Riccati equations will be used in the sequel, [26, Lemma 7 and Theorem 6] and [32, Lemma 8 p. 629].

- If the pair $(A, B)$ is stabilizable and $H$ has no purely imaginary eigenvalues, $\operatorname{CARE}(\Sigma)$ has a unique solution $P^{+}=\left(P^{+}\right)^{\top} \geq 0$ which is stabilising, i.e., all the eigenvalues of $A_{P}$ have strictly negative real part, and $P^{+}$is maximal among the solutions of $\operatorname{CARE}(\Sigma){ }^{1}$

- If $(A, B)$ is anti-stabilizable and $H$ has no eigenvalues on the imaginary axis, $\operatorname{CARE}(\Sigma)$ has a unique solution $P^{-}=\left(P^{-}\right)^{\top} \leq 0$ which is anti-stabilising, i.e., all the eigenvalues of $A_{P}$ have strictly positive real part, and $P^{-}$is minimal among the solutions of the $\operatorname{CARE}(\Sigma)$.

- If $(A, B)$ is controllable and $H$ has no eigenvalues on the imaginary axis, the extreme solutions of $\operatorname{CARE}(\Sigma), P^{+}$and $P^{-}$, both exist. The gap matrix $\Delta:=P^{+}-P^{-}>0$ satisfies the identity $A_{P^{+}}=-\Delta^{-1} A_{P^{-}}^{\top} \Delta$.

- If the pair $(A, B)$ is sign-controllable, i.e., if the set of uncontrollable eigenvalues of $A$ does not contain pairs of elements in the form $(\lambda,-\bar{\lambda})$, [29, 19], $\operatorname{CARE}(\Sigma)$ admits an unmixed solution $P$, i.e., a solution such that if $\lambda,-\bar{\lambda} \in \sigma\left(A_{P}\right)$ implies $\mathfrak{R} e(\lambda)=0,[29,28]$. Moreover, given a solution $P=P^{\top}$ of $\operatorname{CARE}(\Sigma)$ and the corresponding closed-loop matrix $A_{P}$ defined in (9), the Lyapunov equation

$$
A_{P} Y+Y A_{P}^{\top}+B R^{-1} B^{\top}=0
$$

has a unique solution $Y=Y^{\top} \in \mathbb{R}^{n \times n}$ if and only if $P$ is strongly unmixed, which means that $A_{P}$ does not contain mirrored pairs, i.e., $\lambda \in \sigma\left(A_{P}\right)$ implies $-\bar{\lambda} \notin \sigma\left(A_{P}\right)$, [14]. ${ }^{2}$

\footnotetext{
${ }^{1}$ Maximal and minimal are here referred to the standard ordering of symmetric matrices, i.e., $M_{1} \geq M_{2}$ if and only if $M_{1}-M_{2} \geq 0$.

${ }^{2}$ Since $A_{P}$ is real, this is equivalent to the fact that $\sigma\left(A_{P}\right)$ does not contain opposite pairs $(\lambda,-\lambda)$. Clearly, if $P$ is unmixed and none of the eigenvalues of $A_{P}$ lay on the imaginary axis, then $P$ is also strongly unmixed. [22, Theorem 5.2.2].
} 
Remark 1 Sign-controllability is the weakest form of controllability: It is weaker than the assumption of reachability, and even than that of stabilisability or anti-stabilisability of the pair $(A, B)$. Indeed, it generically holds even in the extreme case of $B=0$.

\subsection{Solution of sign-controllable RDE}

The following theorem establishes an explicit expression for the solution of the Riccati differential equation with terminal condition under sign-controllability assumption.

Theorem 2 Assume that $(A, B)$ is sign-controllable and $H$ has no eigenvalues on the imaginary axis. Let $P$ be a strongly unmixed solution of $C A R E(\Sigma)$, let $A_{P}$ be given by (9) and $Y$ be the corresponding solution of (10). The matrix $P(t)=\Lambda(t) X^{-1}(t)$, with

$$
\begin{aligned}
& X(t)=e^{-A_{P}(T-t)}\left(I_{n}-Y\left(P-P_{T}\right)\right)+Y e^{A_{P}^{\top}(T-t)}\left(P-P_{T}\right), \\
& \Lambda(t)=P e^{-A_{P}(T-t)}\left(I_{n}-Y\left(P-P_{T}\right)\right)+\left(P Y-I_{n}\right) e^{A_{P}^{\top}(T-t)}\left(P-P_{T}\right),
\end{aligned}
$$

is the solution of $\operatorname{RDP}\left(\Sigma, P_{T}\right)$.

Proof: First, recall that sign-controllability of $(A, B)$ guarantees that an unmixed solution $P$ of $\operatorname{CARE}(\Sigma)$ exists. Moreover, in view of the absence of purely imaginary eigenvalues of $H$, $P$ is also strongly unmixed, since $\sigma\left(A_{P}\right) \subset \sigma(H)$. Let $P$ be a strongly unmixed solution of $\operatorname{CARE}(\Sigma)$, let $A_{P}$ be the corresponding closed-loop system matrix and $Y$ be the corresponding solution of (10). By adapting the proof of Theorem 1 in [14], it is easy to see that the set of trajectories of $\operatorname{HDE}(\Sigma)$ is parameterised in $\Phi, \Psi \in \mathbb{R}^{n \times n}$ as

$$
\left[\begin{array}{l}
X(t) \\
\Lambda(t)
\end{array}\right]=\left[\begin{array}{c}
I_{n} \\
P
\end{array}\right] e^{A_{P} t} \Phi+\left[\begin{array}{c}
Y \\
P Y-I_{n}
\end{array}\right] e^{A_{P}^{\top}(T-t)} \Psi .
$$

By imposing the boundary conditions $X(T)=I_{n}$ and $\Lambda(T)=P_{T}$ on (13), we find $\Phi=e^{-A_{P} T}\left(Y P_{T}-Y P+I_{n}\right)$ and $\Psi=P-P_{T}$. Thus, the corresponding $X(t)$ and $\Lambda(t)$ are given by (11) and (12). Hence, in view of Theorem $1, X(t)$ is invertible for all $t \in(-\infty, T]$, and $P(t)=\Lambda(t) X^{-1}(t)$ is the solution of $\operatorname{RDP}\left(\Sigma, P_{T}\right)$.

Remark 2 Using the technique described in the proof of Theorem 2, it is also possible to solve the Riccati differential equation with an initial condition

$$
P(0)=P_{0}=P_{0}^{\top} \geq 0
$$

instead of the terminal condition (2). In this case it suffices to impose the boundary conditions $X(0)=I_{n}$ and $\Lambda(0)=P_{0}$ on (13). This yields $\Phi=I_{n}-Y\left(P-P_{0}\right)$ and $\Psi=e^{-A_{P}^{\top} T}\left(P-P_{0}\right)$. 
The matrix $P(t)=\Lambda(t) X^{-1}(t)$, with

$$
\begin{aligned}
& X(t)=e^{A_{P} t}\left(I_{n}-Y\left(P-P_{0}\right)\right)+Y e^{-A_{P}^{\top} t}\left(P-P_{0}\right), \\
& \Lambda(t)=P e^{A_{P} t}\left(I_{n}-Y\left(P-P_{0}\right)\right)+\left(P Y-I_{n}\right) e^{-A_{P}^{\top} t}\left(P-P_{0}\right)
\end{aligned}
$$

is the solution of the Riccati differential equation (1) with the initial condition (14). All the other results that are presented in this paper on the solution of the Riccati differential equation with terminal condition can be adapted in a similar way to the case of an initial condition.

\subsection{A sign-controllability form}

When $(A, B)$ is not sign-controllable, two important sign-controllability forms can be defined. The first has the largest possible sign-controllable part. Let $(A, B)$ be in the Kalman controllability form

$$
A=\left[\begin{array}{cc}
A_{u} & 0 \\
A_{u c} & A_{c}
\end{array}\right] \quad \text { and } \quad B=\left[\begin{array}{c}
0 \\
B_{c}
\end{array}\right],
$$

where $\left(A_{c}, B_{c}\right)$ is controllable. Consider a non-singular matrix $T$ such that $T^{-1} A_{u} T$ is in Jordan form, partitioned as $T^{-1} A_{u} T=\operatorname{diag}\left(A_{u}^{\prime}, A_{u}^{\prime \prime}\right)$. The Jordan blocks corresponding to the eigenvalues of $A_{u}$ are divided between $A_{u}^{\prime}$ and $A_{u}^{\prime \prime}$ according to the following rule:

i) the Jordan blocks corresponding to eigenvalues on the imaginary axis are placed in $A_{u}^{\prime}$;

ii) every Jordan block $B_{\lambda}$ corresponding to an eigenvalue $\lambda$ of $A_{u}$ such that $-\lambda$ is not an eigenvalue of $A_{u}$ is included in $A_{u}^{\prime \prime}$;

iii) for each pair of eigenvalues $(\lambda,-\lambda)$ of $A_{u}$, we denote the corresponding Jordan blocks by $B_{\lambda}$ and $B_{-\lambda}$. If $\operatorname{dim}\left(B_{\lambda}\right) \leq \operatorname{dim}\left(B_{-\lambda}\right)$, we include $B_{\lambda}$ in $A_{u}^{\prime}$ and $B_{-\lambda}$ in $A_{u}^{\prime \prime}$; Otherwise, we include $B_{-\lambda}$ in $A_{u}^{\prime}$ and $B_{\lambda}$ in $A_{u}^{\prime \prime}$.

In this way, $A_{u}^{\prime \prime}$ has no pairs of eigenvalues in the form $(\lambda,-\lambda)$, nor eigenvalues with zero real part. Now, changing coordinates with $U:=\operatorname{diag}(T, I)$ gives

$$
U^{-1} A U=\left[\begin{array}{cc}
T^{-1} A_{u} T & 0 \\
A_{u c} T+A_{c} & A_{c}
\end{array}\right]=\left[\begin{array}{cc|c}
A_{u}^{\prime} & 0 & 0 \\
0 & A_{u}^{\prime \prime} & 0 \\
\hline A_{u c}^{\prime} & A_{u c}^{\prime \prime} & A_{c}
\end{array}\right], \quad U^{-1} B=\left[\begin{array}{c}
0 \\
0 \\
\hline B_{c}
\end{array}\right]
$$

where $\left[\begin{array}{ll}A_{u c}^{\prime} & A_{u c}^{\prime \prime}\end{array}\right]:=A_{u c} T+A_{c}$. The pair $\left(\left[\begin{array}{cc}A_{u}^{\prime \prime} & 0 \\ A_{u c}^{\prime \prime} & A_{c}\end{array}\right],\left[\begin{array}{c}0 \\ B_{c}\end{array}\right]\right)$ is sign-controllable, since $\left(A_{c}, B_{c}\right)$ is controllable and $A_{u}^{\prime \prime}$ has no mirrored pairs of eigenvalues with respect to the imaginary axis, nor eigenvalues on the imaginary axis. Notice that this definition of signcontrollability form provides a sign-controllable part with the largest possible size. 
Remark 3 Consider the matrix differential Riccati equation (1). Consider the change of coordinate matrix $T_{s}$ that transforms the pair $(A, B)$ into the sign-controllability form (or in the controllability form). Define the new Popov triple $\Sigma_{s}$ characterised by the matrices $T_{s}^{-1} A T_{s}$, $T_{s}^{-1} B, T_{s}^{\top} Q T, T^{\top} S$ and $R$. It is easy to establish that, given a solution $P_{s}(t)$ of $\operatorname{RDE}\left(\Sigma_{s}\right)$, the matrix $T_{s}^{-\top} P_{s}(t) T_{s}^{-1}$ is a solution of $\operatorname{RDE}(\Sigma)$, and vice-versa. Moreover, in order to obtain the solution $P(t)$ of $\operatorname{RDP}(\Sigma)$ with boundary condition $P(T)=P_{T}$, one can solve $\operatorname{RDP}\left(\Sigma_{s}\right)$ in $P_{s}(t)$, with boundary condition $P_{s}(T)=T_{s}^{\top} P_{T} T_{s}$, and then set $P(t)=T_{s}^{-\top} P_{s}(t) T_{s}^{-1}$.

When $(A, B)$ is not sign-controllable, but the nonsign-controllable part is unobservable from the Popov matrix, a parameterisation of the solutions of $\operatorname{HDE}(\Sigma)$ based on the solutions of standard algebraic equations can still be established. Let $(A, B)$ be in the sign-controllability form as constructed above, i.e.,

$$
A=\left[\begin{array}{cc}
A_{r} & 0 \\
A_{r s} & A_{s}
\end{array}\right] \quad \text { and } \quad B=\left[\begin{array}{c}
0 \\
B_{s}
\end{array}\right],
$$

where $\left(A_{s}, B_{s}\right)$ is sign-controllable; let $n_{s}$ be the order of $A_{s}$, and let $n_{r}:=n-n_{s}$ be the dimension of the residual (i.e., nonsign-controllable) part. Let the nonsign-controllable subsystem be unobservable from the Popov matrix, i.e., $Q$ and $S$ are partitioned accordingly to this basis as $Q=\operatorname{diag}\left(0_{n_{r} \times n_{r}}, Q_{s}\right)$ and $S=\left[\begin{array}{ll}0_{n_{r} \times m} & S_{s}^{\top}\end{array}\right]^{\top}$ and $A_{r s}=0$. If the Hamiltonian matrix referred to the sole sign-controllable part

$$
H_{s}:=\left[\begin{array}{cc}
A_{s}-B_{s} R^{-1} S_{s}^{\top} & -B_{s} R^{-1} B_{s}^{\top} \\
-Q_{s}+S_{s} R^{-1} S_{s}^{\top} & -A_{s}^{\top}+S_{s} R^{-1} B_{s}^{\top}
\end{array}\right]
$$

has no eigenvalues on the imaginary axis, a matrix $P_{s}$ and a matrix $Y_{s}$ exist satisfying the algebraic Riccati equation referred to the sole sign-controllable part

$$
P_{s} A_{s}+A_{s}^{\top} P_{s}-\left(S_{s}+P_{s} B_{s}\right) R^{-1}\left(S_{s}^{\top}+B_{s}^{\top} P_{s}\right)+Q_{s}=0
$$

and the corresponding closed-loop Lyapunov equation

$$
A_{P_{s}} Y_{s}+Y_{s} A_{P_{s}}^{\top}+B_{s} R^{-1} B_{s}^{\top}=0
$$

respectively, where $A_{P_{s}}:=A_{s}-B_{s} R^{-1}\left(S_{s}^{\top}+B_{s}^{\top} P_{s}\right)$. As such, $P=\operatorname{diag}\left(0_{n_{r} \times n_{r}}, P_{s}\right)$ and $Y=\operatorname{diag}\left(0_{n_{r} \times n_{r}}, Y_{s}\right)$ are the solutions of $\operatorname{CARE}(\Sigma)$ and of the corresponding closed-loop Lyapunov equation referred to the complete system, respectively, as can be proved by means of a simple substitution. By using these matrices in (13), we obtain a parameterisation of the set of trajectories of $\operatorname{HDE}(\Sigma)$, and therefore the result established in Theorem 2 can still be applied in this case using $P$ and $Y$ obtained above. The fact that $H_{s}$ has no eigenvalues on the imaginary axis is necessary to ensure that a solution $Y_{s}$ of (18) exists. Indeed, suppose by contradiction 
that $H_{s}$ has an eigenvalue at $i \omega$, with $\omega \in \mathbb{R}$. It follows that $i \omega$ is an eigenvalue of $A_{P_{s}}$, and, as a consequence, it is an eigenvalue of $A_{P_{s}}^{\top}$, as well. Let $v \in \mathbb{C}^{n}$ denote the eigenvector of $A_{P_{s}}^{\top}$ corresponding to such eigenvalue, i.e., $A_{P_{s}} v=i \omega v$. Let $Y_{s}$ be a solution of (18). By premultiplying and postmultiplying (18) by $v^{*}$ and $v$, respectively, we get

$$
-i \omega v^{*} Y_{s} v+v^{*} Y_{s} i \omega v+v^{*} B_{s} R^{-1} B_{s}^{\top} v=v^{*} B_{s} R^{-1} B_{s}^{\top} v=0
$$

which implies that $v$ lies in the null-space of $B_{s}$. Therefore, $v$ lies in the null-space of $A_{s}^{\top}$, as well, and this means that $i \omega$ is an uncontrollable eigenvalue of the pair $\left(A_{s}, B_{s}\right)$. This is a contradiction, since the pair $\left(A_{s}, B_{s}\right)$ is sign-controllable.

\subsection{Stabilisability}

The case where the pair $(A, B)$ is stabilisable is clearly included, as a particular case, under the sign-controllability assumption. In this case, one can choose as strongly unmixed solution of $\operatorname{CARE}(\Sigma)$ the stabilising solution $P^{+}$, and the result in Theorem 2 still applies with $P=P^{+}$and $A_{P}=A_{P^{+}}$. In the case where $(A, B)$ is stabilisable, an alternative expression for the solution of the $\operatorname{RDE}(\Sigma)$ and $\operatorname{RDP}\left(\Sigma, P_{T}\right)$ can be found by resorting to two elements of the lattice of all symmetric solutions of the algebraic Riccati equation restricted to the sole controllable part. This method has the remarkable computational advantage of reducing the order of the involved algebraic Riccati equation, which, being the sole nonlinear matrix equation to be solved in this procedure, is the sole critical element in terms of numerical robustness, see e.g. [3].

Let $\mathcal{R}$ be the reachable subspace from the origin, whose dimension is here denoted by $n_{c}$; let $n_{u}:=n-n_{c}$. Consider a change of coordinate basis matrix $T_{s}$ such that its first $n_{c}$ columns span $\mathcal{R}$. In the new basis, matrices $A, B$ are partitioned as in (15), while $Q$ and $S$ are partitioned as

$$
Q=\left[\begin{array}{cc}
Q_{u} & Q_{u c} \\
Q_{u c}^{\top} & Q_{c}
\end{array}\right] \text { and } S=\left[\begin{array}{c}
S_{u} \\
S_{c}
\end{array}\right]
$$

The strongly unmixed solution $P^{+}$of $\operatorname{CARE}(\Sigma)$ can be partitioned correspondingly as

$$
P^{+}=\left[\begin{array}{cc}
P_{u}^{+} & P_{u c}^{+} \\
\left(P_{u c}^{+}\right)^{\top} & P_{c}^{+}
\end{array}\right]
$$

and it is easily seen that $P_{c}^{+}$is a solution of the algebraic Riccati equation of the sole controllable part

$$
P_{c}^{+} A_{c}+A_{c}^{\top} P_{c}^{+}-\left(S_{c}+P_{c}^{+} B_{c}\right) R^{-1}\left(S_{c}+P_{c}^{+} B_{c}\right)^{\top}+Q_{c}=0 .
$$


On the other hand, since the pair $\left(A_{c}, B_{c}\right)$ appearing in (20) is controllable, the ARE (20) admits an anti-stabilising solution $P_{c}^{-}$, such that $\sigma\left(A_{P_{c}^{+}}\right)=-\sigma\left(A_{P_{c}^{-}}\right)$. Moreover, the gap matrix $\Delta_{c}:=P_{c}^{+}-P_{c}^{-}$is positive definite, and therefore invertible.

Define also the two $n \times n$ matrices $A^{\star}$ and $P^{\star}$ as

$$
A^{\star}:=\left[\begin{array}{cc}
-A_{u}^{\top} & -Q_{u c}^{\top}+S_{u} R^{-1} S_{c}^{\top}-A_{u c}^{\top} P_{c}^{-}+S_{u} R^{-1} B_{c}^{\top} P_{c}^{-} \\
0 & A_{c}-B_{c} R^{-1}\left(B_{c}^{\top} P_{c}^{-}+S_{c}^{\top}\right)
\end{array}\right]
$$

and $P^{\star}:=\operatorname{diag}\left(I_{n_{u}}, P_{c}^{-}\right)$. Finally, let $J:=\operatorname{diag}\left(0_{n_{u} \times n_{u}}, I_{n_{c}}\right)$.

Theorem 3 Assume that $(A, B)$ is stabilisable and $H$ has no eigenvalues on the imaginary axis. The matrix $P(t)=T_{s}^{-\top} \Lambda(t) X^{-1}(t) T_{s}^{-1}$, with

$$
\begin{aligned}
& X(t)=e^{A_{P^{+}} t} \hat{\Phi}+J e^{A^{\star}(t-T)} \hat{\Psi} \\
& \Lambda(t)=P^{+} e^{A_{P^{+}} t} \hat{\Phi}+P^{\star} e^{A^{\star}(t-T)} \hat{\Psi}
\end{aligned}
$$

where

$$
\hat{\Phi}:=e^{-A_{P^{+}} T}\left(I_{n}+\left[\begin{array}{cc}
0 & 0 \\
0 & \Delta_{c}^{-1}
\end{array}\right]\left(T_{s}^{\top} P_{T} T_{s}-P^{+}\right)\right)
$$

and

$$
\hat{\Psi}:=\left[\begin{array}{cc}
I_{n_{u}} & -P_{u c}^{+} \Delta_{c}^{-1} \\
0 & -\Delta_{c}^{-1}
\end{array}\right]\left(T_{s}^{\top} P_{T} T_{s}-P^{+}\right)
$$

is the solution of $\operatorname{RDP}\left(\Sigma, P_{T}\right)$.

Proof: The functions $X, \Lambda:(-\infty, T] \longrightarrow \mathbb{R}^{n \times n}$ defined by

$$
\left[\begin{array}{c}
X(t) \\
\Lambda(t)
\end{array}\right]=\left[\begin{array}{c}
I_{n} \\
P^{+}
\end{array}\right] e^{A_{P^{+}} t} \Phi+\left[\begin{array}{c}
J \\
P^{\star}
\end{array}\right] e^{A^{\star}(t-T)} \Psi
$$

represent a parameterisation of all the solutions of the Hamiltonian differential equation (3) in $\Phi$ and $\Psi$. This fact can be proved by direct substitution of (26) into $\operatorname{HDE}(\Sigma)$ written with all submatrices of $H$ partitioned as in (15) and (19). Moreover, all the solutions of the Hamiltonian differential equation (3) can be expressed through (26) for a suitable choice of $\Phi$ and $\Psi$. In fact, the images of $S_{1}:=\left[\begin{array}{ll}I_{n} & P^{+}\end{array}\right]^{\top}$ and $S_{2}:=\left[\begin{array}{ll}J & P^{\star}\end{array}\right]^{\top}$ are $H$-invariant subspaces, since it can be proved by direct substitution that $H S_{1}=S_{1} A_{P^{+}}$and $H S_{2}=S_{2} A^{\star}$. The eigenvalues of $H$ restricted to $i m S_{1}$ coincide with the eigenvalues of $A_{P^{+}}$, while the eigenvalues of $H$ restricted to $\mathrm{im} S_{2}$ are the eigenvalues of $A^{\star}$. However, from the discussion above it follows that the eigenvalues of $A_{P^{+}}$and those of $A^{\star}$ are opposite, i.e., $\sigma\left(A_{P^{+}}\right)=-\sigma\left(A^{\star}\right)$. As a result of this, $i m S_{1} \cap i m S_{2}=\{0\}$, which implies that (26) represents a set of $2 n^{2}$ linearly independent 
trajectories of $\operatorname{HDE}(\Sigma)$. To find the solution of $\operatorname{RDP}\left(\Sigma, P_{T}\right)$, we need to find the matrices $\Phi$ and $\Psi$ to be used in (26) so that (26) represents the solution of $\operatorname{HDP}\left(\Sigma, P_{T}\right)$. By imposing the boundary conditions in (26) as indicated in Remark 3, we get $e^{A_{P^{+}}{ }^{T}} \Phi+J \Psi=I_{n}$ and $P^{+} e^{A_{P^{+}} T} \Phi+P^{\star} \Psi=T_{s}^{\top} P_{T} T_{s}$. Matrix $\Phi$ can be computed from the first and substituted into the second, to get

$$
\left(P^{\star}-P J\right) \Psi=T_{s}^{\top} P_{T} T_{s}-P^{+} .
$$

Matrix $P^{\star}-P^{+} J$ can be written as

$$
P^{\star}-P^{+} J=\left[\begin{array}{cc}
I_{n_{u}} & -P_{u c}^{+} \\
0 & P_{c}^{-}-P_{c}^{+}
\end{array}\right],
$$

and is easily seen to be invertible, and yields

$$
\Psi=\left[\begin{array}{cc}
I_{n_{u}} & -P_{u c}^{+} \Delta_{c}^{-1} \\
0 & -\Delta_{c}^{-1}
\end{array}\right]\left(T_{s}^{\top} P_{T} T_{s}-P^{+}\right) .
$$

By substition of this value of $\Psi$ into $\Phi=e^{-A_{P^{+}} T}\left(I_{n}-J \Psi\right)$, we get (24).

\section{Dropping the sign-controllablity assumption}

As already observed, sign-controllability is a very weak and general system-theoretic assumption. Nevertheless, this assumption could still result to be restrictive in some situations. Therefore, in this section we show that some results can be extended even when the signcontrollability assumption does not hold. In other terms, we investigate a further generalisation of the results obtained for sign-controllable pairs can be achieved under milder assumptions, that do not guarantee the existence of a solution of $\operatorname{CARE}(\Sigma)$ and of the corresponding Lyapunov equation. To the best of the authors' knowledge, sign-controllability is the weakest assumption on the pair $(A, B)$ for which $\operatorname{CARE}(\Sigma)$ is guaranteed to admit a symmetric solution. Therefore, without the sign-controllability assumptions, $\operatorname{CARE}(\Sigma)$ might not admit a symmetric solution. The parameterisation of the trajectories of $\operatorname{HDE}(\Sigma)$ involves the (strongly unmixed) solution of the algebraic Riccati equation restricted to the sole sign-controllable part of the system. However, the sign-controllability form needed here must be constructed in a different way than that presented in Section 3.3, as will become clear in Theorem 4.

Let $(A, B)$ be in the Kalman controllability canonical form (15). Define $\Lambda:=\sigma\left(A_{u}\right) \cap \sigma\left(-A_{u}\right)$. There exists $T \in \mathbb{R}^{n \times n}$ such that $T^{-1} A_{u} T=\operatorname{diag}\left(A_{u}^{\prime}, A_{u}^{\prime \prime}\right)$ with $\sigma\left(A_{u}^{\prime}\right)=\Lambda$. Changing coordinates with $U:=\operatorname{diag}(T, I)$ gives (16) where, again, the pair $\left(\left[\begin{array}{cc}A_{u}^{\prime \prime} & 0 \\ A_{u c}^{\prime \prime} & A_{c}\end{array}\right],\left[\begin{array}{c}0 \\ B_{c}\end{array}\right]\right)$ is signcontrollable. Notice that, differently from the sign-controllability form introduced in Section 
3.3, in this case for each pair of uncontrollable eigenvalues the corresponding Jordan blocks $B_{\lambda}$ and $B_{-\lambda}$ are both placed in the nonsign-controllable part. Hence, the size of the resulting sign-controllable part is smaller than that obtained using the sign-controllability form as defined in Section 3.3. Now, let the pair $(A, B)$ be in this second sign-controllability form,

$$
A=\left[\begin{array}{cc}
A_{r} & 0 \\
A_{r s} & A_{s}
\end{array}\right] \quad \text { and } \quad B=\left[\begin{array}{c}
0 \\
B_{s}
\end{array}\right],
$$

where the pair $\left(A_{s}, B_{s}\right)$ is sign-controllable, and let $Q$ and $S$ be partitioned accordingly, i.e.,

$$
Q=\left[\begin{array}{cc}
Q_{r} & Q_{r s} \\
Q_{r s}^{\top} & Q_{s}
\end{array}\right] \quad \text { and } S=\left[\begin{array}{c}
S_{r} \\
S_{s}
\end{array}\right]
$$

Moreover, define $F_{s r}:=A_{r s}-B_{s} R^{-1} S_{r}^{\top}, F_{s s}:=A_{s}-B_{s} R^{-1} S_{s}^{\top}, G_{r}:=-Q_{r}+S_{r} R^{-1} S_{r}^{\top}$, $G_{s}:=-Q_{s}+S_{r} R^{-1} S_{s}^{\top}, G_{r s}=-Q_{r s}+S_{s} R^{-1} S_{s}^{\top}$ and $V:=-B_{s} R^{-1} B_{s}^{\top}$. With respect to this basis, the Hamiltonian differential equation can be written as

$$
\left[\begin{array}{c}
\dot{X}_{r}(t) \\
\dot{X}_{s}(t) \\
\dot{\Lambda}_{r}(t) \\
\dot{\Lambda}_{s}(t)
\end{array}\right]=\bar{H}\left[\begin{array}{c}
X_{r}(t) \\
X_{s}(t) \\
\Lambda_{r}(t) \\
\Lambda_{s}(t)
\end{array}\right] \text {, where } \bar{H}=\left[\begin{array}{cccc}
A_{r} & 0 & 0 & 0 \\
F_{s r} & F_{s s} & 0 & V \\
G_{r} & G_{s} & -A_{r}^{\top} & -F_{s r}^{\top} \\
G_{s}^{\top} & G_{r s} & 0 & -F_{s s}^{\top}
\end{array}\right] .
$$

In the former, the time varying matrices $X(t)$ and $\Lambda(t)$ have been partitioned according to the sign-controllability form. The Hamiltonian matrix $H_{s}$ referred to the sole sign-controllable part is

$$
H_{s}=\left[\begin{array}{cc}
F_{s s} & V \\
G_{r s} & -F_{s s}^{\top}
\end{array}\right] .
$$

In the next theorem, it is shown that if $A_{r}$ and $H_{s}$ have no common eigenvalues, and if $H_{s}$ has no eigenvalues on the imaginary axis, a characterisation of the solutions of $\operatorname{HDE}(\Sigma)$ is still possible. Notice that if instead of the sign-controllability form defined in this section we used that presented in Section 3.3, for each pair $(\lambda,-\lambda)$ of uncontrollable eigenvalues, the corresponding Jordan blocks $B_{\lambda}$ and $B_{-\lambda}$ would be divided between the sign-controllable and the nonsign-controllable part. As a result, the sign-controllable part would have uncontrollable eigenvalues that are opposite in sign to some nonsign-controllable eigenvalues. However, since uncontrollable eigenvalues and their opposites are also eigenvalues of the Hamiltonian matrix, it follows that the spectrum of the Hamiltonian matrix of the sign-controllable part $H_{s}$ would include the pair $(\lambda,-\lambda)$, and therefore it would end up having eigenvalues in common with the nonsign-controllable part $A_{r}$. As a result, the Sylvester equations used in the next theorem to construct the parameterisation of the trajectories of $\operatorname{HDE}(\Sigma)$ would not be guaranteed to admit solutions. 
Theorem 4 Let the Popov triple be in the second sign-controllability form, as defined in this section. Assume $\sigma\left(A_{r}\right) \cap \sigma\left(H_{s}\right)=\emptyset$ and that $H_{s}$ has no eigenvalues on the imaginary axis. Let $U_{1}, U_{2} \in \mathbb{R}^{n_{r} \times n_{s}}$ be given by the solution of the Sylvester equation

$$
A_{r}^{\top}\left[\begin{array}{ll}
U_{1} & U_{2}
\end{array}\right]+\left[\begin{array}{ll}
U_{1} & U_{2}
\end{array}\right] H_{s}=\left[\begin{array}{ll}
G_{s} & -F_{s r}^{\top}
\end{array}\right],
$$

and let $U_{3}, U_{4} \in \mathbb{R}^{n_{r} \times n_{s}}$ be given by the solution of the Sylvester equation

$$
-A_{r}^{\top}\left[\begin{array}{ll}
U_{3} & U_{4}
\end{array}\right]+\left[\begin{array}{ll}
U_{3} & U_{4}
\end{array}\right] H_{s}^{\top}=-\left[\begin{array}{ll}
F_{s r}^{\top} & G_{s}
\end{array}\right] .
$$

The set of trajectories originating from $\operatorname{HDE}(\Sigma)$ is parameterised in $\Gamma \in \mathbb{R}^{2 n_{r} \times n}, \Phi \in \mathbb{R}^{n_{s} \times n}$ and $\Psi \in \mathbb{R}^{n_{s} \times n}$ as

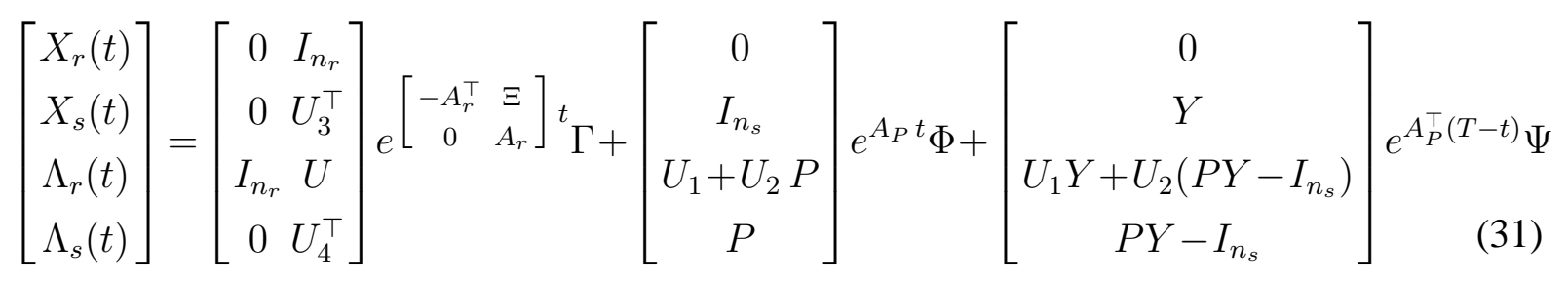

where $\Xi:=-U_{1} F_{s r}+G_{r}-U_{2} G_{s}^{\top}$ and $U:=U_{1} U_{3}^{\top}+U_{2} U_{4}^{\top}$.

Proof: We construct three changes of coordinates for the Hamiltonian differential equation, in order to obtain a block-diagonal structure for $H$. Since $\sigma\left(A_{r}\right) \cap \sigma\left(H_{s}\right)=\emptyset$, the Sylvester equation (29) admits a unique solution $\left[\begin{array}{ll}U_{1} & U_{2}\end{array}\right]$. Consider the non-singular matrix

$$
T_{1}=\left[\begin{array}{cccc}
I_{n_{r}} & 0 & 0 & 0 \\
0 & 0 & I_{n_{s}} & 0 \\
0 & I_{n_{r}} & U_{1} & U_{2} \\
0 & 0 & 0 & I_{n_{s}}
\end{array}\right], \text { so that } T_{1}^{-1}=\left[\begin{array}{cccc}
I_{n_{r}} & 0 & 0 & 0 \\
0 & -U_{1} & I_{n_{s}} & -U_{2} \\
0 & I_{n_{r}} & 0 & 0 \\
0 & 0 & 0 & I_{n_{s}}
\end{array}\right] .
$$

By taking (29) into account, we get

$$
H_{1}:=T_{1}^{-1} \bar{H} T_{1}=\left[\begin{array}{cccc}
A_{r} & 0 & 0 & 0 \\
\Xi & -A_{r}^{\top} & 0 & 0 \\
F_{s r} & 0 & F_{s s} & V \\
G_{s}^{\top} & 0 & G_{r s} & -F_{s s}^{\top}
\end{array}\right]
$$

where $\Xi:=-U_{1} F_{s r}+G_{r}-U_{2} G_{s}^{\top}$. Since $\sigma\left(A_{r}\right) \cap \sigma\left(H_{s}\right)=\emptyset$, the Sylvester equation (30) admits a unique solution $\left[\begin{array}{cc}U_{3} & U_{4}\end{array}\right]$. Consider the non-singular matrix

$$
T_{2}=\left[\begin{array}{cccc}
0 & I_{n_{r}} & 0 & 0 \\
I_{n_{r}} & 0 & 0 & 0 \\
0 & U_{3}^{\top} & I_{n_{s}} & 0 \\
0 & U_{4}^{\top} & 0 & I_{n_{s}}
\end{array}\right], \text { so that } T_{2}^{-1}=\left[\begin{array}{cccc}
0 & I_{n_{r}} & 0 & 0 \\
I_{n_{r}} & 0 & 0 & 0 \\
-U_{3}^{\top} & 0 & I_{n_{s}} & 0 \\
-U_{4}^{\top} & 0 & 0 & I_{n_{s}}
\end{array}\right] \text {. }
$$


The new change of coordinates over $H_{1}$ yields

$$
H_{2}=T_{2}^{-1} H_{1} T_{2}=\left[\begin{array}{cccc}
-A_{r}^{\top} & \Xi & 0 & 0 \\
0 & A_{r} & 0 & 0 \\
0 & 0 & F_{s s} & V \\
0 & 0 & G_{r s} & -F_{s s}^{\top}
\end{array}\right],
$$

which is block-diagonal. A further change of coordinates is performed using

$T_{3}=\left[\begin{array}{cccc}0 & I_{n_{r}} & 0 & 0 \\ I_{n_{r}} & 0 & 0 & 0 \\ 0 & 0 & I_{n_{s}} & 0 \\ 0 & 0 & 0 & I_{n_{s}}\end{array}\right]$, so that $H_{3}=T_{3}^{-1} H_{2} T_{3}=\left[\begin{array}{cc|cc}A_{r} & 0 & 0 & 0 \\ \Xi & -A_{r}^{\top} & 0 & 0 \\ \hline 0 & 0 & F_{s s} & V \\ 0 & 0 & G_{r s} & -F_{s s}^{\top}\end{array}\right]$,

The submatrix in the right-bottom of $H_{3}$ is exactly $H_{s}$. Now, consider the Hamiltonian differential equation in this new basis:

$$
\left[\begin{array}{c}
\dot{X}_{1}(t) \\
\dot{\Lambda}_{1}(t) \\
\dot{X}_{2}(t) \\
\dot{\Lambda}_{2}(t)
\end{array}\right]=H_{3}\left[\begin{array}{c}
X_{1}(t) \\
\Lambda_{1}(t) \\
X_{2}(t) \\
\Lambda_{2}(t)
\end{array}\right]
$$

where

$$
\left[\begin{array}{c}
X_{r}(t) \\
X_{s}(t) \\
\Lambda_{r}(t) \\
\Lambda_{s}(t)
\end{array}\right]=T_{1} T_{2} T_{3}\left[\begin{array}{c}
X_{1}(t) \\
\Lambda_{1}(t) \\
X_{2}(t) \\
\Lambda_{2}(t)
\end{array}\right]=\left[\begin{array}{c}
X_{1}(t) \\
U_{3}^{\top} X_{1}(t)+X_{2}(t) \\
U X_{1}(t)+\Lambda_{1}(t)+U_{1} X_{2}(t)+U_{2} \Lambda_{2}(t) \\
U_{4}^{\top} X_{1}(t)+\Lambda_{2}(t)
\end{array}\right] .
$$

Since the pair $\left(A_{s}, B_{s}\right)$ is sign-controllable and $H_{s}$ has no eigenvalues on the imaginary axis, we can compute the strongly unmixed solution of the sole sign-controllable part $P$, the closedloop matrix and the solution of the corresponding closed-loop Lyapunov equation $A_{P}$ and $Y$, respectively. Hence, due to the block-diagonal structure of $H_{3}$, the parameterisation of the solutions of the Hamiltonian differential equation in the new basis is decoupled as

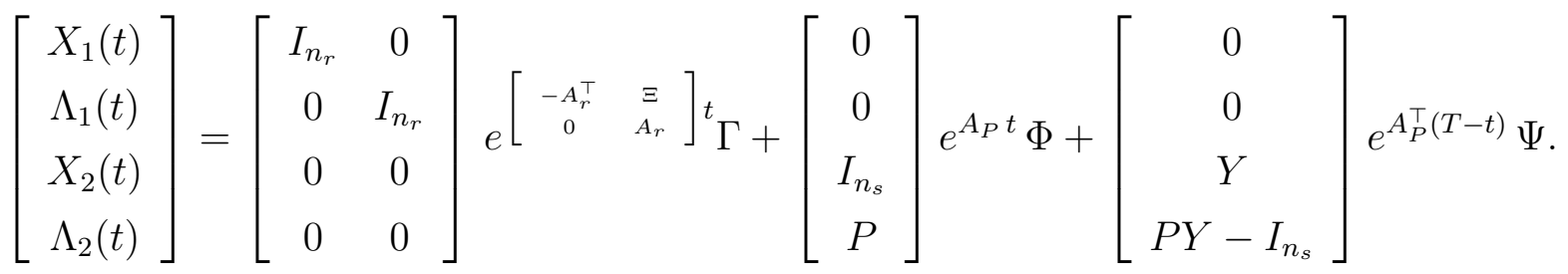

Writing this equation with respect to the original basis gives (31).

Our aim is now to show how the result established in Theorem 4 can be exploited to find a representation of the solution of the Riccati differential equation with terminal condition $\operatorname{RDP}\left(\Sigma, P_{T}\right)$. The solution to this problem is given in the following theorem. 
Theorem 5 Let $T_{s}$ be a change of basis matrix that transforms the pair $(A, B)$ into the second sign-controllability form as defined in Section 4, and let matrices $Q$ and $S$ be partitioned as in (28). Assume $\sigma\left(A_{r}\right) \cap \sigma\left(H_{s}\right)=\emptyset$ and that $H_{s}$ has no eigenvalues on the imaginary axis. Let $U_{1}, U_{2}, U_{3}, U_{4} \in \mathbb{R}^{n_{r} \times n_{s}}$, be the solutions of (29) and (30), respectively. Let

$$
\left[\begin{array}{c}
X_{r}(T) \\
X_{s}(T) \\
\Lambda_{r}(T) \\
\Lambda_{s}(T)
\end{array}\right]=\left[\begin{array}{c}
M_{r} \\
M_{s} \\
N_{r} \\
N_{s}
\end{array}\right], \text { where }\left[\begin{array}{c}
M_{r} \\
M_{s}
\end{array}\right]=I_{n} \text { and }\left[\begin{array}{c}
N_{r} \\
N_{s}
\end{array}\right]=T_{s}^{\top} P_{s} T_{s} .
$$

The solution $P(t)$ of $R D P(\Sigma)$ is given by

$$
P(t)=T_{s}^{-\top}\left[\begin{array}{l}
\Lambda_{r}(t) \\
\Lambda_{s}(t)
\end{array}\right]\left[\begin{array}{l}
X_{r}(t) \\
X_{s}(t)
\end{array}\right]^{-1} T_{s}^{-1},
$$

where $X_{s}(t), X_{r}(t), \Lambda_{s}(t)$ and $\Lambda_{r}(t)$ are given by (31) with

$$
\left[\begin{array}{c}
\Phi \\
\Psi \\
\Gamma_{1} \\
\Gamma_{2}
\end{array}\right]=\left[\begin{array}{cccc}
e^{-A_{P} T}(I-Y P) & e^{-A_{P} T} Y & 0 & 0 \\
P & -I & 0 & 0 \\
U_{1} & \left(U_{1}+U_{2} P\right)(I-Y)+U_{2} & -\Omega-e^{A_{r} T} U & e^{A_{r} T} \\
0 & 0 & e^{-A_{r} T} & 0
\end{array}\right]\left[\begin{array}{c}
M_{s}-U_{3}^{\top} M_{r} \\
N_{s}-U_{4}^{\top} M_{r} \\
M_{r} \\
N_{r}
\end{array}\right] .
$$

Proof: Consider $\operatorname{HDP}\left(\Sigma, P_{T}\right)$, and consider the change of coordinate matrix $T_{s}$ that takes the original Popov triple into the second sign-controllability form. By imposing (32) on $X_{r}$ and $X_{s}$ in (31) we obtain

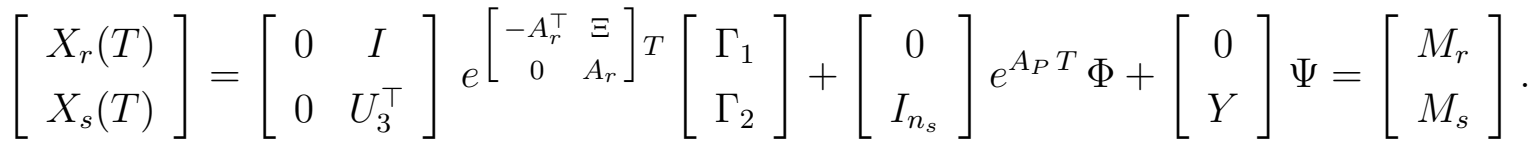

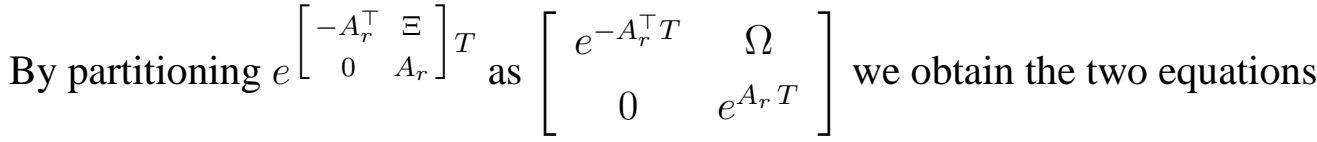

$$
\left[\begin{array}{c}
e^{A_{r} T} \Gamma_{2} \\
U_{3}^{\top} e^{A_{r} T} \Gamma_{2}+e^{A_{P} T} \Phi+Y \Psi
\end{array}\right]=\left[\begin{array}{c}
M_{r} \\
M_{s}
\end{array}\right]
$$

which lead to $\Gamma_{2}=e^{-A_{r} T} M_{r}$ and

$$
\left[\begin{array}{cc}
e^{A_{P} T} & Y
\end{array}\right]\left[\begin{array}{l}
\Phi \\
\Psi
\end{array}\right]=M_{s}-U_{3}^{\top} M_{r} .
$$

By imposing the boundary condition on $\Lambda_{r}$ and $\Lambda_{s}$ in (31) we obtain

$$
\begin{aligned}
{\left[\begin{array}{c}
\Lambda_{r}(T) \\
\Lambda_{s}(T)
\end{array}\right]=} & {\left[\begin{array}{cc}
I & U \\
0 & U_{4}^{\top}
\end{array}\right]\left[\begin{array}{cc}
e^{-A_{r}^{\top} T} & \Omega \\
0 & e^{A_{r} T}
\end{array}\right]\left[\begin{array}{c}
\Gamma_{1} \\
\Gamma_{2}
\end{array}\right]+\left[\begin{array}{c}
U_{1}+U_{2} P \\
P
\end{array}\right] e^{A_{P} T} \Phi } \\
& +\left[\begin{array}{c}
U_{1} Y+U_{2}(P Y-I) \\
P Y-I
\end{array}\right] \Psi=\left[\begin{array}{c}
N_{r} \\
N_{s}
\end{array}\right]
\end{aligned}
$$


which leads to

$$
\left[\begin{array}{c}
e^{-A_{r} T} \Gamma_{1}+\left(\Omega+U e^{A_{r} T}\right) \Gamma_{2}+\left(U_{1}+U_{2} P\right) e^{A_{P} T} \Phi+\left[U_{1} Y+U_{2}(P Y-I)\right] \Psi \\
U_{4}^{\top} e^{A_{r} T} \Gamma_{2}+P e^{A_{P} T} \Phi+(P Y-I) \Psi
\end{array}\right]=\left[\begin{array}{c}
N_{r} \\
N_{s}
\end{array}\right]
$$

The first block-equation enables $\Gamma_{1}$ to be computed given the values of $\Phi, \Psi$ and $\Gamma_{2}$. The second gives

$$
P e^{A_{P} T} \Phi+(P Y-I) \Psi=N_{s}-U_{4}^{\top} M_{r}
$$

From (33) and (34) we obtain

$$
\left[\begin{array}{cc}
e^{A_{P} T} & Y \\
P e^{A_{P} T} & P Y-I
\end{array}\right]\left[\begin{array}{c}
\Phi \\
\Psi
\end{array}\right]=\left[\begin{array}{c}
M_{s}-U_{3}^{\top} M_{r} \\
N_{s}-U_{4}^{\top} M_{r}
\end{array}\right]
$$

The Schur complement of the first matrix on the left hand side $(P Y-I)-P e^{A_{P} T} e^{-A_{P} T} Y=$ $-I$ is invertible. As such, (35) can be inverted:

$$
\begin{aligned}
{\left[\begin{array}{l}
\Phi \\
\Psi
\end{array}\right] } & =\left[\begin{array}{cc}
e^{A_{P} T} & Y \\
P e^{A_{P} T} & P Y-I
\end{array}\right]^{-1}\left[\begin{array}{c}
M_{s}-U_{3}^{\top} M_{r} \\
N_{s}-U_{4}^{\top} M_{r}
\end{array}\right] \\
& =\left[\begin{array}{cc}
e^{-A_{P} T}(I-Y P) & e^{-A_{P} T} Y \\
P & -I
\end{array}\right]\left[\begin{array}{c}
M_{s}-U_{3}^{\top} M_{r} \\
N_{s}-U_{4}^{\top} M_{r}
\end{array}\right]
\end{aligned}
$$

and

$$
\begin{aligned}
\Gamma_{1}= & e^{A_{r} T}\left(N_{r}-\left(\Omega e^{-A_{r} T}+U\right) M_{r}+\left(U_{1}+U_{2} P\right) e^{A_{P} T} \Phi+\left[U_{1} Y+U_{2}(P Y-I)\right] \Psi\right) \\
= & e^{A_{r} T}\left(N_{r}-\left(\Omega e^{-A_{r} T}+U\right) M_{r}+U_{1}\left(M_{s}-U_{3}^{\top} M_{r}\right)\right. \\
& \left.+\left(U_{1}+U_{2} P-U_{1} Y-U_{2} P Y+U_{2}\right)\left(N_{s}-U_{4}^{\top} M_{r}\right)\right) .
\end{aligned}
$$

\section{Applications to LQ optimal control}

In this section, we investigate how the material developed in this paper, and in particular in Section 4, can be utilised to solve very general LQ optimal control problems. In particular, in [14] it was shown how to use parameterised expressions of the trajectories generated by the Hamiltonian differential equation to solve the most general form of LQ optimal control problem with affine constraints on the state vector at the end points and with a quadratic performance index; in [14] this very general problem is tackled under the assumption of sign-controllability. Here we show how to use the results of Section 4 to cases where the pair $(A, B)$ might not 
be sign-controllable. For the sake of argument, however, here we restrict our attention to the so-called fixed end-point LQ problem. Consider the LTI state differential equation

$$
\dot{x}(t)=A x(t)+B u(t),
$$

where, for all $t \geq 0, x(t) \in \mathbb{R}^{n}$ is the state, $u(t) \in \mathbb{R}^{m}$ is the control input. Moreover, it is required that

$$
\begin{aligned}
x(0) & =\bar{x}_{0} \in \mathbb{R}^{n} . \\
x(T) & =\bar{x}_{T} \in \mathbb{R}^{n} .
\end{aligned}
$$

The problem here is to find a measurable $u(t), t \in[0, T)$, and an absolutely continuous $x(t)$, $t \in[0, T]$, minimising the quadratic performance index

$$
J(x, u):=\int_{0}^{T}\left[x^{\top}(t) u^{\top}(t)\right] \Pi\left[\begin{array}{l}
x(t) \\
u(t)
\end{array}\right] d t,
$$

under the constraints (36) and (37-38). We show how the result in Theorem 4 can be utilised to the end of constructing the optimal control law. For the sake of simplicity, we consider the system to be already in the sign-controllable form, so that no preliminary coordinate changes are needed. If $u(t)$ and $x(t)$ are optimal for the fixed end-point LQ problem, then a costate function $\lambda(t) \in \mathbb{R}^{n}$ exists such that $x(t), \lambda(t)$ and $u(t)$ satisfy for all $t \in[0, T)$ the following set of equations:

$$
\begin{aligned}
{\left[\begin{array}{c}
\dot{x}(t) \\
\dot{\lambda}(t)
\end{array}\right] } & =\left[\begin{array}{cc}
A-B R^{-1} S^{\top} & -B R^{-1} B^{\top} \\
-Q+S R^{-1} S^{\top} & -A^{\top}+S R^{-1} B^{\top}
\end{array}\right]\left[\begin{array}{l}
x(t) \\
\lambda(t)
\end{array}\right], \\
u(t) & =-R^{-1}\left(S^{\top} x(t)+B^{\top} \lambda(t)\right), \\
x(0) & =\bar{x}_{0}, \\
x(T) & =\bar{x}_{T},
\end{aligned}
$$

These equations follow as a particular case of those in [14, Lemma 1]. Equation (40) differs from the Hamiltonian differential equation (3) only for the dimension of the unknown vector. As such, the set of all the trajectories satisfying (40) is given by (31), where now $\Phi, \Psi$ and $\Gamma$ are vectors. By partitioning $\bar{x}_{0}=\left[\begin{array}{cc}\bar{x}_{r 0}^{\top} & \bar{x}_{s 0}^{\top}\end{array}\right]^{\top}$ and $\bar{x}_{T}=\left[\begin{array}{cc}\bar{x}_{r T}^{\top} & \bar{x}_{s T}^{\top}\end{array}\right]^{\top}$ conformably with the sign-controllability form, by imposing (42-43) on (40) we get

$$
\left[\begin{array}{c}
\Gamma_{2} \\
U_{3}^{\top} \Gamma_{2}+\Phi+Y e^{A_{P}^{\top} T} \Psi
\end{array}\right]=\left[\begin{array}{c}
\bar{x}_{r 0} \\
\bar{x}_{s 0}
\end{array}\right]
$$

and

$$
\left[\begin{array}{c}
e^{A_{r} T} \Gamma_{2} \\
U_{3}^{\top} e^{A_{r} T} \Gamma_{2}+e^{A_{P} T} \Phi+Y \Psi
\end{array}\right]=\left[\begin{array}{c}
\bar{x}_{r T} \\
\bar{x}_{s T}
\end{array}\right]
$$


By eliminating $\Gamma_{2}$ from these two equations we obtain the condition $\bar{x}_{r T}=e^{A_{r} T} \bar{x}_{r 0}$, which is not surprising. In fact, the fixed end-point problem can be solved only if the nonsigncontrollable part of the state, which is obviously also uncontrollable, reaches the target $\bar{x}_{r T}$ starting from the initial value $\bar{x}_{r 0}$ by itself. The remaining equations lead to the linear relation

$$
\left[\begin{array}{cc}
I & Y e^{A_{P}^{\top} T} \\
e^{A_{P} T} & Y
\end{array}\right]\left[\begin{array}{c}
\Phi \\
\Psi
\end{array}\right]=\left[\begin{array}{c}
\bar{x}_{s 0}-U_{3}^{\top} \bar{x}_{r 0} \\
\bar{x}_{s T}-U_{3}^{\top} \bar{x}_{r 0}
\end{array}\right]
$$

which cannot be inverted in general. In fact, within the sign-controllable subsystem there are in general uncontrollable modes. Therefore, the fact that the condition $\bar{x}_{r T}=e^{A_{r} T} \bar{x}_{r 0}$ is satisfied does not guarantee that the fixed end-point LQ problem admits solutions, because there are other uncontrollable modes in the sign-controllable part. Therefore, the $2 n \times 2 n$ matrix in the left-hand side of (44) is not invertible in general. Hence, the other condition that needs to be satisfied for the LQ problem to be solvable is that

$$
\left[\begin{array}{c}
\bar{x}_{s 0}-U_{3}^{\top} \bar{x}_{r 0} \\
\bar{x}_{s T}-U_{3}^{\top} \bar{x}_{r 0}
\end{array}\right] \in \operatorname{im}\left[\begin{array}{cc}
I & Y e^{A_{P}^{\top} T} \\
e^{A_{P} T} & Y
\end{array}\right] .
$$

If this condition holds true, the remaining parameters $\Phi$ and $\Psi$ can be determined by pseudoinversion of (44). These values of $\Phi, \Psi, \Gamma_{2}$ (and with arbitrary $\Gamma_{1}$ ) can be used in (31), which at that point provides the trajectory of (40) satisfying (42) and (43). Then, the state and costate can be replaced in (41) to provide a control law which is optimal for the LQ control problem at hand, since $x(t), \lambda(t)$ and $u(t)$ satisfy all the necessary and sufficient conditions required to guarantee optimality.

\section{Concluding remarks}

The formulae established in this paper for the solution of the matrix Riccati differential equation, which generalise those proposed so far in the literature, can be used to extend the analysis of finite escape time of the solution of the Riccati differential equation carried out in [4, p.1240]. Moreover, important properties of the dynamic behaviour of the solution of $\operatorname{RDE}(\Sigma)$, such as convergence and mechanisms of attraction, which have been studied in the literature under the assumptions of controllability and stabilisability, can be generalised using the results presented here.

\section{References}

[1] B.D.O. Anderson and J.B. Moore. Optimal Control: Linear Quadratic Methods. Prentice Hall International, London, 1989. 
[2] S. Bittanti, A.J. Laub and J.C. Willems. The Riccati Equation. Springer-Verlag, Berlin, 1991.

[3] P. Benner, and P. Van Dooren. Advanced Computational Tools for Computer-Aided Control System Design (CACSD). Tutorial Workshop in European Control Conference 2003, Cambridge (UK), September 1 - 4, 2003.

[4] F.M. Callier and J.L. Willems. Large finite horizon and infinite horizon LQ-optimal control problems. Optimal Control: Applications and Methods, 4:31-45, 1983.

[5] F.M. Callier, J.J. Winkin, and J.L. Willems. Convergence of the time-invariant Riccati differential equation and LQ-problem: mechanisms of attraction. International Journal of Control, 59(4):983-1000, 1994.

[6] F.M. Callier and J.J. Winkin. Asymptotic behavious of the solution of the projection Riccati differential equation. IEEE Transactions on Automatic Control, AC-41(5):646659, 1996.

[7] P. Colaneri, and A. Ferrante, "Algebraic Riccati equation and J-spectral factorization for H-infinity estimation ”. Systems \& Control Letters, 51/5: 383-393, 2004.

[8] P. Colaneri and A. Ferrante. Algebraic Riccati equation and $J$-spectral factorization for $\mathcal{H}_{\infty}$ filtering and deconvolution. SIAM J. Contr. and Opt.. Vol. 45, No. 1, pp. 123-145, 2006.

[9] W.A. Coppel. Linear-quadratic optimal control. In Proceedings of the Royal Society of Edinburgh, volume 73A, pages 271-289, 1974.

[10] E.J. Davison and M.C. Maki. The numerical solution of the matrix Riccati differential equation. IEEE Transactions on Automatic Control, AC-18(1):71-73, 1973.

[11] Y. Feng and B.D.O. Anderson An iterative algorithm to solve periodic Riccati differential equations with an indefinite quadratic term. In Proceedings of the Conference on Decision and Control 2008, Cancun, Mexico, 2008.

[12] A. Ferrante, and L. Ntogramatzidis. A unified approach to finite-horizon generalized LQ optimal control problems for discrete-time systems. Linear Algebra and its Applications (Special Issue in honor of Paul Fuhrmann), Vol. 425, pp. 242-260, 2007.

[13] A. Ferrante, and L. Ntogramatzidis. Employing the algebraic Riccati equation for a parametrization of the solutions of the finite-horizon LQ problem: the discrete-time case. System \& Control Letters. Vol. 54(7):693-703, May 2005. 
[14] A. Ferrante, and L. Ntogramatzidis, "A Unified Approach to the Finite-Horizon Linear Quadratic Optimal Control Problem”. European Journal of Control, 13/5: 473-488, 2007.

[15] A. Ferrante, G. Marro, and L. Ntogramatzidis. A parametrization of the solutions of the finite-horizon LQ problem with general cost and boundary conditions. Automatica, 1(8):1359-1366, August 2005.

[16] V. Ionescu, C. Oară, and M. Weiss. Generalized Riccati theory and robust control, a Popov function approach. Wiley, 1999.

[17] S. Johansson, B. Kågstr"om, A. Shiriaev and A. Varga. Comparing one-shot and multishot methods for solving periodic Riccati differential equations. In Proceedings of the IFAC Workshop on Periodic Control Systems, St. Petersburg, Russia, 2007.

[18] C.S. Kenney and R.B. Leipnik. Numerical integration od the Differential Matrix Riccati Equation. IEEE Transactions on Automatic Control, AC-30(10):962-970, 1985.

[19] V. Kučera. Algebraic Riccati equations: Hermitian and definite solutions. in: S. Bittanti et al. (Eds.), The Riccati Equation, Communications and Control Engeneering, Springer, Berlin, 1991.

[20] H. Kwakernaak and R. Sivan. Linear Optimal Control Systems. John Wiley \& Sons, New York, 1972.

[21] D.G. Lainiotis. Generalized Chandrasekhar algorithms: Time-varying models. IEEE Transactions on Automatic Control, AC-20, p.728, 1976.

[22] P. Lancaster and L. Rodman. Algebraic Riccati equations. Clarendon Press, Oxford, 1995.

[23] F.L. Lewis and V. Syrmos. Optimal Control. John Wiley \& Sons, New York, 1995.

[24] G. Marro, L. Ntogramatzidis, and E. Zattoni. $H_{2}$-Optimal Decoupling of Previwed Signals in the Continuous-Time Domain. In Proceedings of the 2004 American Control Conference (2004 ACC), pages 2717-2722, Boston, Massachussets (USA), June 30 - July 2, 2004.

[25] W.M. McEneaney. A new fundamental solution for differential Riccati equations arising in control. Automatica, 44:920-936, 2008.

[26] B.P. Molinari. The time-invariant linear-quadratic optimal control problem. Automatica, 13:347-357, 1977. 
[27] L. Ntogramatzidis, and G. Marro, "A parametrization of the solutions of the Hamiltonian system for stabilizable pairs". International Journal of Control, 78(7): 530-533, May 2005.

[28] C. Scherer. The solution set of the algebraic Riccati Equation and the Algebraic Riccati Inequality. Linear Algebra and Its Applications, 153:99-122, 1991.

[29] M.A. Shayman. Geometry of the algebraic Riccati equation, Part I. SIAM Journal of Control and Optimization, 21:375-394, 1983.

[30] A. Varga. On solving periodic Riccati equations. Numerical Linear Algebra with Applications, 15(9):809-835, 2008.

[31] A. Varga. On solving periodic differential matrix equations with applications to periodic system norms computation. In Proceedings of the Conference on Decision and Control 2005, Seville, Spain, 2005.

[32] J.C. Willems. Least squares stationary optimal control and the algebraic Riccati equation. IEEE Transactions on Automatic Control, AC-16(6):621-634, 1971. 